\title{
Incorporating time-resolved three-dimensional phase contrast (4D flow) MRI in clinical workflow: initial experiences at a large tertiary care medical center
}

\author{
Bradley D Allen*, Alex J Barker, Keyur Parekh, Lewis C Sommerville, Susanne Schnell, Kelly B Jarvis, Maria Carr, \\ James Carr, Jeremy Collins, Michael Markl \\ From 16th Annual SCMR Scientific Sessions \\ San Francisco, CA, USA. 31 January - 3 February 2013
}

\section{Background}

Time-resolved three-dimensional phase contrast (4D flow) MRI allows for visualization of three-dimensional cardiovascular anatomy and pulsatile flow with full volumetric coverage in a single, easy to prescribe 3D acquisition. The technique provides comprehensive flow visualization and permits retrospective flow quantification at any user-defined region of interest. [1] To our knowledge, no center has incorporated 4D flow MRI as a part of standard clinical cardiovascular MRI (CMR). The goals of this study include: 1 ) reporting on the incorporation of 4D flow MRI acquisition and processing as part of clinical CMR workflow and 2) better understanding the clinical impact of 3D flow visualization and retrospective flow quantification derived from 4D flow MRI in CMR.

\section{Methods}

Patients referred to Northwestern Memorial Hospital for CMR with relevant clinical indications as judged by an attending radiologist were selected to have $4 \mathrm{D}$ flow MRI included in accordance with an IRB-approved protocol. Images were processed using in-house software for noise reduction, anti-aliasing, and eddy current correction. Flow visualization and quantification were performed using EnSight (CEI, Apex, NC). Processing time was recorded. Resulting 4D flow pathline cine images and flow quantification results were converted to DICOM format and transferred to the local PACS server to be reviewed as part of the patient's clinical images.

\footnotetext{
Radiology, Northwestern University Feinberg School of Medicine, Chicago, IL,
} USA

C 2013 Allen et al; licensee BioMed Central Ltd. This is an Open Access article distributed under the terms of the Creative Commons Attribution License (http://creativecommons.org/licenses/by/2.0), which permits unrestricted use, distribution, and reproduction in any medium, provided the original work is properly cited.
Clinician-requested quantitative data was compared between $4 \mathrm{D}$ flow and two-dimensional phase contrast (2D PC) MRI techniques. Each case was retrospectively reviewed by an attending radiologist who assigned a qualitative measure of the 4D flow analysis' impact on the case 1) excluding 2D PC and 2) including 4D flow and 2D PC together (criteria listed in Table 1).

\section{Results}

Sixteen patients had clinical 4D flow MRI over 10 weeks. Clinical indications are reviewed in Table 1 . The average 4D flow impact factor (scale 1-4) excluding and including 2D PC was $2.93+/-0.77$ and $2.80+/-0.75$, respectively. The average percent difference in quantitative data was $35+/-28 \%$. One patient could not be evaluated secondary to aliasing. Average time for $4 \mathrm{D}$ flow post-processing was $88.5+/-22.5 \mathrm{~min}$. Three clinical cases are shown in Figure 1.

\section{Conclusions}

Including 4D flow MRI as part of clinical CMR workflow is feasible and has the potential to impact clinical assessment in multiple cardiovascular pathologies. The ability to evaluate flow throughout the acquired 3D volume retrospectively may reduce dependence on timeintensive 2D PC acquisitions while yielding accurate and efficient flow quantification.

\section{Funding}

Grant support: NIH R01HL115828, NUCATS Dixon Award. 
Table 1 Clinical cases with 4D flow imaging requests and results of analysis.

\begin{tabular}{|c|c|c|c|c|c|c|c|c|}
\hline & & Quantitative $r$ & esults & & & & \multicolumn{2}{|c|}{$\begin{array}{c}\text { Subjective 4D Flow Impact Factor (scale: } \\
\text { changed clinical impression }=4 \text {, quantitative } \\
\text { information added }=3 \text {, relevant but did not } \\
\text { impact case }=2 \text {, information not relevant to } \\
\text { case }=1 \text { ) }\end{array}$} \\
\hline & & Clinical indication for $4 \mathrm{D}$ flow request & $\begin{array}{l}\text { Quantitative } \\
\text { comparison } \\
\text { requested }\end{array}$ & $4 \mathrm{D}$ results & $2 \mathrm{D} P C$ results & Difference (\%) & $\begin{array}{c}\text { 4D flow alone } \\
\text { (excludes } 2 \mathrm{D} \text { PC } \\
\text { data) }\end{array}$ & $\begin{array}{l}\text { Case including both } 2 \mathrm{D} \\
\mathrm{PC} \text { and } 4 \mathrm{D} \text { flow }\end{array}$ \\
\hline & 1 & Biscuspid aortic valve, flow pattern interest & $\begin{array}{l}\text { Regurgitant } \\
\text { fraction }\end{array}$ & $16.57 \%$ & $25 \%$ & $34 \%$ & 3 & 2 \\
\hline & 2 & Qp:Qs ratio in patient with VSD & Qsp:Qs ratio & 1.22 & $\begin{array}{c}\text { Not } \\
\text { measured }\end{array}$ & N/A & 3 & 3 \\
\hline & 3 & Aortic stenosis, flow pattern interest & $\begin{array}{l}\text { Aortic root } \\
\text { peak velocity }\end{array}$ & $3.52 \mathrm{~m} / \mathrm{s}$ & $3.8 \mathrm{~m} / \mathrm{s}$ & $7 \%$ & 2 & 3 \\
\hline & 4 & Biscuspid valve, flow pattern interest & $\begin{array}{l}\text { Aortic root } \\
\text { peak velocity }\end{array}$ & $2.35 \mathrm{~m} / \mathrm{s}$ & $\begin{array}{l}\text { Note } \\
\text { measured }\end{array}$ & N/A & 3 & 2 \\
\hline \multirow[t]{4}{*}{ Valvular } & 5 & $\begin{array}{l}\text { Biscuspid valve, flow pattern interest, aortic regurgitation. } \\
\text { Regurgitant jet is very eccentric, limiting } 2 \mathrm{D} \text { assessment }\end{array}$ & $\begin{array}{l}\text { Regurgitant } \\
\text { fraction }\end{array}$ & $13.36 \%$ & $57 \%$ & $77 \%$ & 2 & 3 \\
\hline & 6 & $\begin{array}{c}\text { Biscuspid aortic valve, flow pattern interest, aortic regurgitation } \\
\text { and stenosis assessment }\end{array}$ & $\begin{array}{l}\text { Regurgitant } \\
\text { fraction }\end{array}$ & $8.75 \%$ & $34 \%$ & $74 \%$ & 2 & 3 \\
\hline & 7 & Aortic root velocity, 2D PC data underestimated velocity & $\begin{array}{l}\text { Aortic root } \\
\text { peak velocity }\end{array}$ & $4.08 \mathrm{~m} / \mathrm{s}$ & $3.5 \mathrm{~m} / \mathrm{s}$ & $17 \%$ & 4 & 4 \\
\hline & 8 & $\begin{array}{l}\text { Bicuspid aortic valve, flow pattern interest, aortic regurgitation and } \\
\text { stenosis assessment }\end{array}$ & $\begin{array}{l}\text { Regurgitant } \\
\text { fraction }\end{array}$ & $4.66 \%$ & $15 \%$ & $69 \%$ & 3 & 2 \\
\hline \multirow[t]{4}{*}{ Aneurysm } & 9 & Flow pattern interest, aortic dilation & $\begin{array}{c}\text { Ascending } \\
\text { aorta peak } \\
\text { velocity }\end{array}$ & $1.44 \mathrm{~m} / \mathrm{s}$ & $1.3 \mathrm{~m} / \mathrm{s}$ & $11 \%$ & 3 & 2 \\
\hline & 10 & Aortic stenosis and regurgitation assessment & $\begin{array}{l}\text { Regurgitant } \\
\text { fraction }\end{array}$ & $38 \%$ & $55 \%$ & $31 \%$ & 3 & 3 \\
\hline & & & $\begin{array}{l}\text { Aortic root } \\
\text { peak velocity }\end{array}$ & $3.36 \mathrm{~m} / \mathrm{s}$ & $3.8 \mathrm{~m} / \mathrm{s}$ & $12 \%$ & & \\
\hline & 11 & Aortic stenosis and regurgitation assessment & $\begin{array}{l}\text { Aortic root } \\
\text { peak velocity }\end{array}$ & $3.89 \mathrm{~m} / \mathrm{s}$ & $4.0 \mathrm{~m} / \mathrm{s}$ & $5 \%$ & 4 & 4 \\
\hline
\end{tabular}


Table 1 Clinical cases with 4D flow imaging requests and results of analysis. (Continued)

\begin{tabular}{|c|c|c|c|c|c|c|c|c|}
\hline & 12 & Flow pattern interest, split flow right-left PA & $\begin{array}{l}\text { Peak velocity } \\
\text { through } \\
\text { pulmonary } \\
\text { anastomis }\end{array}$ & $2.32 \mathrm{~m} / \mathrm{s}$ & $1.4 \mathrm{~m} / \mathrm{s}$ & $66 \%$ & 4 & 4 \\
\hline & & & $\begin{array}{l}\text { Peak velocity } \\
\text { through } \\
\text { pulmonary } \\
\text { post-stenosis }\end{array}$ & $2.70 \mathrm{~m} / \mathrm{s}$ & $2.5 \mathrm{~m} / \mathrm{s}$ & $8 \%$ & & \\
\hline \multirow[t]{3}{*}{$\begin{array}{c}\text { Post- } \\
\text { surgical }\end{array}$} & 13 & Aortic valve assessment & $\begin{array}{c}\text { Ascending } \\
\text { aorta peak } \\
\text { velocity }\end{array}$ & $\begin{array}{l}\text { Could not } \\
\text { measure } \\
\text { velocity } \\
\text { secondary } \\
\text { to aliasing } \\
\text { artifact }\end{array}$ & $\begin{array}{c}\text { Not } \\
\text { measured }\end{array}$ & N/A & Not available & Not available \\
\hline & 14 & Aortic stenosis and regurgitation assessment & $\begin{array}{l}\text { Regurgitant } \\
\text { fraction }\end{array}$ & $4.53 \%$ & $\begin{array}{c}\text { Data limited } \\
\text { by artifact }\end{array}$ & N/A & 3 & 3 \\
\hline & & & $\begin{array}{l}\text { Aortic root } \\
\text { peak velocity }\end{array}$ & $3.11 \mathrm{~m} / \mathrm{s}$ & $\begin{array}{c}\text { Data limited } \\
\text { by artifact }\end{array}$ & N/A & & \\
\hline \multirow[t]{4}{*}{ Congenital } & 15 & Large pulmonary regurgitant fraction by $2 \mathrm{D} P C$ & $\begin{array}{l}\text { Pulmonary } \\
\text { valve } \\
\text { regurgitant } \\
\text { fraction }\end{array}$ & $6.41 \%$ & $21.5 \%$ & $70 \%$ & 1 & 2 \\
\hline & 16 & Flow pattern, Qp:Qs & Qp:Qs Ratio & 1.08 & 1 & $8 \%$ & 3 & 3 \\
\hline & Average & $35 \%$ & 2.93 & 2.80 & & & & \\
\hline & St dev & $28.31 \%$ & 0.77 & 0.75 & & & & \\
\hline
\end{tabular}




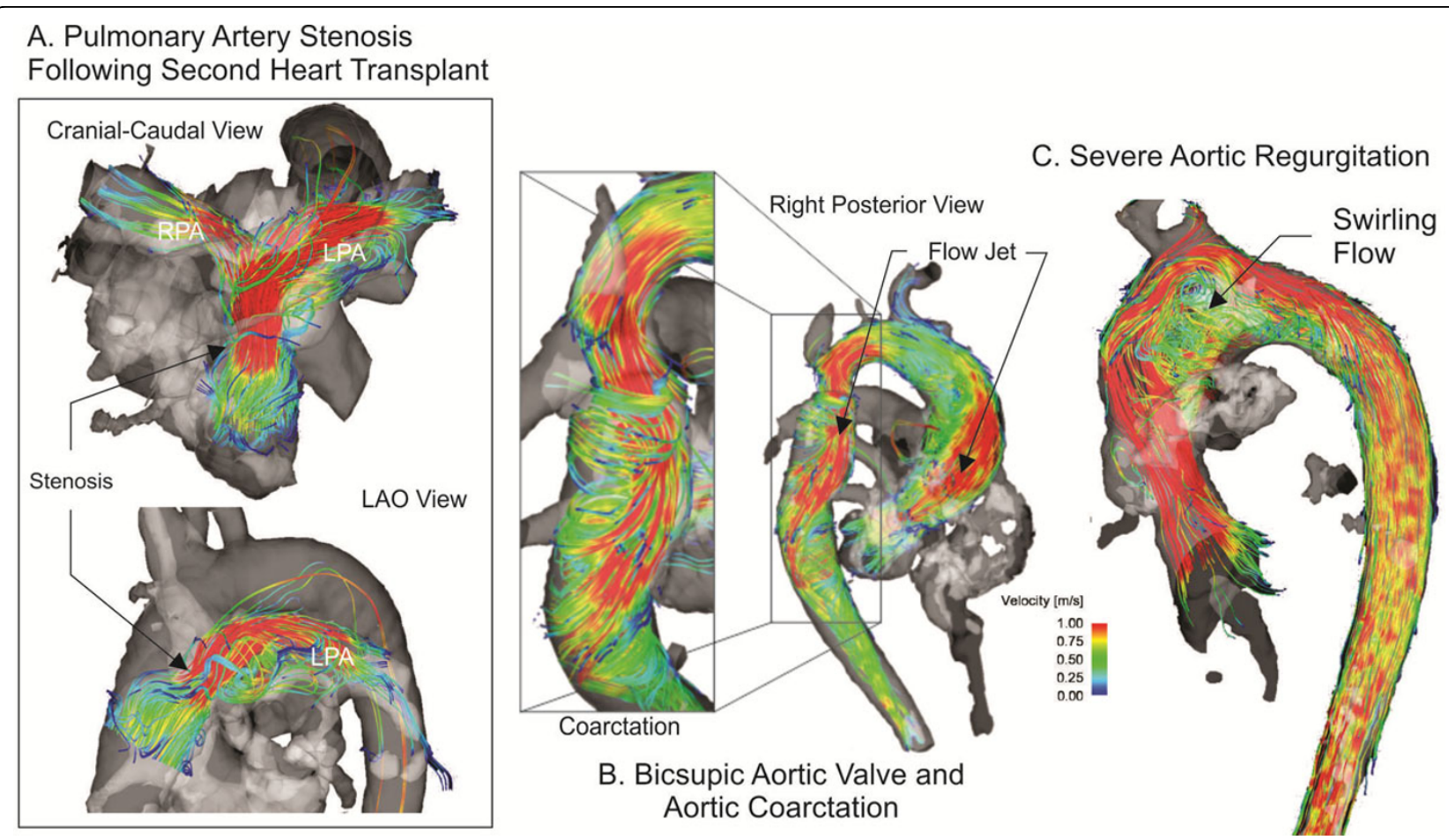

Figure 1 Velocity streamline flow representation in three clinical cases. A. Abnormal pulmonary flow in patient with pulmonary artery stenosis after two heart transplants. Note the helical flow and high velocities after the stenosis directed toward the left pulmonary artery (LPA) and decreased flow towards the right pulmonary artery (RPA). B. Deranged flow along the entire thoracic aorta secondary to bicuspid aortic valve and aortic coarctation. Note high velocity flow jet directed posteriorly with helix formation in the ascending aorta, and high velocity flow jet with helix formation distal to the coarctation. C. Flow abnormalities secondary to severe congenital aortic insufficiency. Note the high velocity systolic flow jet accompanied by swirling flow in the ascending aorta resulting from large volume aortic regurgitation.

\section{Reference}

1. Markl M, et al.. J Cardiovasc MR 2011, 13:7.

\section{Submit your next manuscript to BioMed Central} and take full advantage of:

- Convenient online submission

- Thorough peer review

- No space constraints or color figure charges

- Immediate publication on acceptance

- Inclusion in PubMed, CAS, Scopus and Google Scholar

- Research which is freely available for redistribution 